\title{
La contribution de Friedrich Wilhelm Zahn (1845-1904) à l'étude de l'inflammation
}

par Lazare Benaroyo

\section{RÉsumé}

Avant d'être appelé à occuper la première chaire de pathologie à l'Université de Genève, Friedrich Wilhelm Zahn a publié en 1871 sa thèse de doctorat intitulée: Zur Lehre von der Entzündung und Eiterung. Ce travail, rédigé sous la direction d'Edwin Klebs, à Berne, démontre expérimentalement que le prétendu Microsporon septicum (un champignon infectieux identifié par ce dernier) provoque une inflammation locale ainsi que l'apparition de foyers de suppuration secondaires, à distance. Cette étude confirme apparemment l'hypothèse de Klebs selon laquelle le Microsporon septicum, habituellement présent dans les plaies, est la cause d'une maladie infectieuse. La recherche de Zahn fait d'emblée l'objet d'une critique, également adressée à Klebs : l'identification du microorganisme, de même que la démonstration de son action causale ne sont pas concluantes. A l'issue du débat, il est admis qu'un microorganisme ne peut être la cause d'un état infectieux qu'aux deux conditions suivantes: il doit être considéré comme identifié selon la méthode de Koch, et la relation causale qui l'unit à la maladie infectieuse doit être spécifique. C'est ce que la thèse de Zahn n'est précisément pas parvenue à démontrer.

L'analyse du contenu de cette recherche ainsi que des critiques qu'elle a suscitées nous permet de mettre en lumière les divers arguments qui ont alimenté la controverse sur la cause de l'inflammation et de la suppuration à l'aube de l'ère bactériologique.

Il est une question qui a fait couler beaucoup d'encre autour des années 1870 : quelle est la cause de la suppuration?

La formation du pus, telle que nous la concevons actuellement, est habituellement attribuée à une cause bactérienne: il s'agit d'une infection de l'organisme par une bactérie appartenant le plus fréquemment au genre micrococcus, de la famille des staphylocoques ou de celle des streptocoques. ${ }^{1}$ Cette théorie représente pour nous, depuis les années 1880, une certitude sur laquelle se base une importante partie de la thérapeutique chirurgicale et 
médicale; elle se fonde sur des observations ainsi que des conceptualisations qui, avant d'être acceptées sous la forme que nous leur connaissons, ont fait l'objet entre les années 1870 et 1880 d'une instructive controverse qui mérite, à mon sens, que l'on y prête une attention particulière.

Les comptes rendus annuels des divers arguments avancés de part et d'autre au cours du débat ont été fidèlement retranscrits dans les Schmidt's Jahrbücher der in- und ausländischen gesammten Medicin ainsi que dans les Jahresberichte über die Leistungen und Fortschritte in der gesammten Medicin. ${ }^{2}$ Nous y trouvons mentionnés de nombreux auteurs tels le botaniste de Breslau Ferdinand Cohn (1828-1898), le chirurgien viennois Theodor Billroth (1829-1894), le collègue et ami de Claude Bernard, Casimir Davaine (1812-1882), l'anatomiste et pathologiste Victor Feltz (1835-1893), le pharmacologue Léon Coze (1817-1896) de même que plusieurs pathologues allemands tels Wilhelm Waldeyer (1836-1921), Carl Weigert (1845-1904), sans compter les nombreux élèves de Rudolf Virchow (1821-1902): Georg Eduard Rindfleisch (1836-1908), Friedrich Daniel von Recklinghausen (1833-1910), Julius Cohnheim (1839-1884), Edwin Klebs (1834-1913) ainsi que son élève Friedrich Wilhelm Zahn (1845-1904). ${ }^{3}$

Il peut paraître surprenant de trouver cité dans le cadre de cette controverse le nom de F. W. Zahn. Ce dernier est en effet plus habituellement connu des pathologues contemporains pour ses travaux sur la constitution microscopique du thrombus : c'est à lui que l'on doit en effet la description des trois formes de thrombi - le thrombus blanc, rouge et mixte - qui date de $1875 ;{ }^{4}$ il était à cette époque l'assistant de F. D. von Recklinghausen à Strasbourg. Il est de même reconnu pour ses recherches sur la pathogenèse de l'infarctus hémorragique (qui porte son nom) ${ }^{5}$, ainsi que pour ses travaux sur la nature des modifications myocardiques consécutives à l'insuffisance aortique. ${ }^{6}$ Ces deux dernières études ont été entreprises alors qu'il occupait, depuis 1876, le premier poste de professeur de pathologie à l'Université de Genève. ${ }^{7}$

Zahn débute sa carrière médicale par des études successivement effectuées à Erlangen, à Heidelberg (il y suit l'enseignement de H. Helmholtz), puis à Berne; c'est là qu'il devient l'assistant du pathologue Edwin Klebs. ${ }^{8} \mathrm{Il}$ rédige sa thèse de doctorat sous sa direction; le travail est publié en 1871 à Heidelberg sous le titre suivant: Zur Lehre von der Entzündung und Eiterung. Mit besonderer Berücksichtigung der durch das Mikrosporon septicum hervorgerufenen Erscheinungen. Cette recherche, peu connue, mérite d'être étudiée dans le détail: elle se situe en effet au cœur du débat qui nous occupe.

Edwin Klebs, qui est mobilisé en 1870 comme médecin militaire à 
Karlsruhe, est frappé par le fait que sur les 115 autopsies qu'il pratique, $73 \%$, soit 84 cas, présentent une pyémie ou une septicémie. La septicémie témoigne assurément du caractère infectieux de la maladie. L'observation microscopique des sécrétions purulentes des plaies (sur le vivant), ainsi que des multiples foyers d'embolisation septique (à l'autopsie) montre la présence constante d'un microorganisme. Il s'agit d'un champignon polymorphe qu'il dénomme le Microsporon septicum.

Lidentification est faite selon la méthode récemment mise au point par F. D. von Recklinghausen : ${ }^{9}$ ses caractéristiques optiques, sa résistance aux alcalis et aux acides, ainsi que son comportement face aux colorants permettent de lui attribuer ce nom. Il est considéré comme un vrai champignon constitué par un mycélium et des spores de taille et de forme variables; certaines formes peuvent d'ailleurs prendre l'aspect d'une bactérie. La doctrine du pléomorphisme, alors en vogue, admet la genèse d'une bactérie à partir d'un champignon souche. ${ }^{10}$ Klebs est convaincu qu'il doit exister une relation de causalité entre ce microorganisme et la suppuration tant locale (au niveau des plaies) que générale (la septicémie), observées chez les blessés. Comment cependant démontrer que le Microsporon septicum est la cause d'une maladie infectieuse?

Il soumet cette démonstration à deux exigences : 1) Il faut démontrer que le champignon est présent dans les plaies suppurées, qu'il pénètre ensuite dans les tissus et provoque des foyers de suppurations secondaires, c'est-àdire une septicémie; 2) Il faut isoler l'agent causal et démontrer son action pathogène sur l'organisme. Il s'agit-là de «l'experimentum crucis». Klebs procède à la démonstration du premier point $\mathrm{il}$ constate que la présence du Microsporon septicum est toujours accompagnée de phénomènes suppuratifs; ces phénomènes sont à la fois locaux et généraux. Des thrombophlébites ainsi que des foyers emboliques métastatiques sont observés: cela lui permet d'émettre l'idée que ce champignon est très probablement la cause de la septicémie. Si tel est bien le cas, le Microsporon septicum doit également être la cause de la suppuration primaire constatée au niveau des plaies: il doit donc être la cause d'une infection locale qui se propage de proche en proche et provoque ainsi le développement d'une infection générale secondaire.

C'est précisément la vérification de cette hypothèse qui est soumise à Zahn. La recherche expérimentale alors menée par ce dernier aboutit à la conclusion que le Microsporon septicum isolé, puis inoculé à la grenouille, peut provoquer une suppuration locale, qui peut se généraliser et devenir dans certains cas létale. 
Les deux exigences étant ainsi remplies, l'action pathogène du champignon suggerée par Klebs est confirmée. Ces résultats sont publiés dans un article intitulé: «Die Ursache der infectiösen Wundkrankheiten» paru dans le premier volume du Correspondenz-Blatt für schweizer Ärzte de 1871. Une communication plus détaillée des observations est publiée en 1872 dans les Beiträge zur pathologischen Anatomie der Schusswunden nach Beobachtungen in den Kriegslazarethen in Karlsruhe. Klebs cite dans ces textes les recherches de Zahn.

Les critiques qui paraissent la même année, portent essentiellement sur les prémisses posées par Klebs, et grèvent par là même les conclusions de Zahn. Felix Birch-Hirschfeld (1842-1889), alors «Prosektor» à Dresde, estime dans son compte rendu paru en 1872 dans les Schmidt's Jahrbücher, ${ }^{11}$ que le travail de Klebs, - qui selon lui marquera une époque, - comporte cependant une importante faiblesse: l'identification du microorganisme n'est pas assez bien établie; la description rappelle en effet des formes de champignons inférieurs déjà décrites par d'autres auteurs. Theodor Billroth, alors professeur de chirurgie à Vienne, estime de même, dans son article publié dans les Archiv für klinische Chirurgie de 1872, ${ }^{12}$ que les preuves avancées par Klebs ne sont pas suffisantes pour considérer le Microsporon septicum comme l'agent causal de la suppuration. Les observations morphologiques ne sont en effet pas convaincantes et les altérations tissulaires attribuées au microorganisme pourraient être causées par des facteurs différents, tel par exemple un produit chimique, - quand bien même celui-ci serait sécrété par ce champignon. Pour être valide, la démonstration de Klebs devrait prouver, selon Billroth, que le Microsporon septicum est le principal, si ce n'est le seul agent capable de causer une suppuration, ce que ce travail ne met pas en évidence. Cette critique pleine de sagacité semble d'autant plus légitime qu'elle survient la même année où le botaniste F. Cohn, farouche adversaire de la doctrine du pléomorphisme, propose une classification des bactéries fondée sur des critères à la fois morphologiques et fonctionnels permettant de les différencier des champignons. ${ }^{13}$ Le Microsporon septicum étant constitué de formes de passage appartenant tant aux bactéries qu'aux champignons pourrait bien n'être qu'une chimère!

Moins hardi que Klebs, Billroth reste prudent dans l'interprétation de l'action pathogène de la Coccobacteria septica, une algue constituée de formes végétatives d'allure bactérienne et micrococcique qu'il découverte: il constate sa présence lors de phénomènes suppuratifs, mais ne conclut pas pour d'autant à une relation de cause à effet. ${ }^{14}$ 
Les débats se poursuivent ainsi jusqu'en $1880,{ }^{15}$ quand Alexander Ogston (1844-1929), chirurgien à Aberdeen, parvient à démontrer qu'une bactérie du genre micrococcus est responsable de la formation du pus dans les abcès aigus. ${ }^{16} \mathrm{Il}$ s'agit-là de la première étude sur laquelle se fondent nos certitudes actuelles.

Mais revenons aux années 1870, et penchons-nous sur la thèse de Zahn; étudions la place qu'elle occupe au sein de la controverse qui nous intéresse; examinons plus en détail, après les avoir placés dans leur contexte, les arguments positifs qu'il avance pour démontrer que le Microsporon septicum est l'agent causal de la suppuration. C'est alors seulement que nous pourrons évaluer avec le recul critique nécessaire, l'intérêt historique de cette recherche.

Pour Zahn, comme pour bon nombre de ses collègues, la suppuration et l'inflammation ont acquis, suite aux recherches de Julius Cohnheim, une identité génétique. En 1867, en effet, alors qu'il est à Berlin l'assistant de Rudolf Virchow et le collègue de laboratoire de F. D. von Recklinghausen, ${ }^{17}$ Cohnheim démontre expérimentalement sur le mésentère de la grenouille que l'émigration active des globules blancs à travers la paroi des vaisseaux capillaires veineux constitue le processus de l'inflammation aiguë. ${ }^{18}$ Les éléments cellulaires de l'exsudat inflammatoire, les cellules du pus, dont l'origine a fait l'objet de nombreuses controverses durant les deux premiers tiers du $19^{\mathrm{e}}$ siècle, ${ }^{19}$ sont en fait des globules blancs venus du sang. ${ }^{20}$

Si les faits établis par Cohnheim permettent de trouver une explication à des observations morphologiques antérieures, ils n'en soulèvent pas moins quelques interrogations. ${ }^{21}$ L'assistant berlinois utilise par exemple indifféremment l'air, le coton ou des insectes comme stimuli expérimentaux. ${ }^{22}$ Peut-on en conclure que l'inflammation aiguë connait diverses causes? Cette question, qui ne semble pas retenir son attention à cette époque, ${ }^{23}$ fait l'objet d'une série d'investigations: quelle est la cause de l'émigration des globules blancs?

Parmi ses confrères pathologues qui cherchent à résoudre ce problème, ${ }^{24}$ Edwin Klebs risque en 1871 une hypothèse: un agent biologique pourrait-il se substituer aux divers facteurs chimiques habituellement proposés ? ${ }^{25} \mathrm{Le}$ Microsporon septicum identifié dans les préparations microscopiques des sécrétions des plaies des blessés de la guerre franco-prussienne pourrait-il être cet agent? ${ }^{26}$

Il incombe alors à Zahn de compléter les recherches de Klebs et d'examiner si ce champignon, une fois isolé, peut engendrer de manière reproductible 
une émigration des globules blancs à travers la paroi des vaisseaux. Avant de procéder à la démonstration, l'assistant bernois décrit avec grand soin le matériel et les méthodes utilisés.

Son étude porte sur des observations pratiquées sur le mésentère de la grenouille au préalable curarisée. ${ }^{27}$ Cet organe est mis à nu, puis placé entre une lame et une lamelle: il évite ainsi de devoir le fixer avec des aiguilles, sources de phénomènes irritatifs. La préparation est étudiée dans un premier temps directement sous le microscope. Elle est ensuite placée dans un appareil permettant de modifier la nature de l'air ambiant.

L'instrument optique utilisé est un microscope performant, de marque Hartnack; des mesures sont effectuées avec un oculaire spécial. La première série d'expériences utilise l'air comme stimulus et teste son effet sur la dilatation des vaisseaux et l'émigration des globules blanc à travers leurs parois.

Lorsque l'air ambiant est filtré, grâce à l'appareil décrit ci-dessus, à travers du coton ou de l'acide phénique, aucune émigration n'est observée. Ce fait est attesté par une étude portant sur 10 cas; dans les 3 cas d'exsudation tardive relevée après 24 heures, des microorganismes divers (vibrions, bactéries, champignons) ont été identifiés; leur présence a été considérée comme concomitante au phénomène inflammatoire. ${ }^{28}$

Cette première série d'expérimentations permet à Zahn de démontrer que l'air purifié ne provoque aucune inflammation. ${ }^{29} \mathrm{Il}$ lui est alors possible de déduire que l'air à son état naturel doit contenir des substances pyogènes (dénommées Fremdkörper dans le texte). Le fait qu'il puisse s'agir de microorganismes est supposé, car l'action antiseptique de l'acide phénique est bien connue à cette époque : ${ }^{30}$

«Reine Luft wirkt nicht eiterungserregend, sondern die Auswanderung wird durch ihr beigemengte, aber daraus entfernbare Fremdkörper hervorgerufen.» ${ }^{31}$

La deuxième série d'expériences utilise comme stimulus un liquide infectant, prélevé d'un abcès pulmonaire d'un patient décédé de pyémie, et contenant le Microsporon septicum (identifié d'après la méthode de Klebs). Le contenu du prélèvement, filtré à plusieurs reprises à travers de la terre d'argile, ${ }^{32}$ laisse un résidu contenant le champignon; ce dernier est alors mis en culture sur un milieu constitué de tartrate d'ammoniaque: un contrôle microscopique atteste sa présence dans le liquide ainsi obtenu.

Zahn teste dans un premier temps l'effet de l'application directe de ce liquide sur le mésentère. L'étude porte sur 10 cas. L'émigration des globules 
blancs, ainsi que l'éventuelle constatation à l'autopsie de la généralisation du phénomène suppuratif sont retenues et comparées, dans chaque cas, à un témoin non infecté. Les conclusions sont les suivantes:

«Das Microsporon septicum beschleunigt und steigert die Auswanderung in hohem Masse; es verursacht selbst den Tod der Untersuchungsthiere.

Dasselbe gelangt von der Wundfläche aus in den Organismus.» ${ }^{33}$

Il teste dans un deuxième temps l'effet d'un filtrat du liquide, exempt du champignon. ${ }^{34}$ L'étude porte sur 5 cas, examinés comme précédemment en comparaison à un temoin. L'expérience démontre que le filtrat a un pouvoir pyogène, déjà à faible dose:

«Es scheint von diesem Pilz ein für den Organismus höchst schädliches Gift produziert zu werden, das in dem Filtrat der Pilzflüssigkeit enthalten ist.

Dasselbe wird in geringerer Menge vom Organismus ertragen und die so behandelten Thiere können am Leben bleiben, während es in grösseren Mengen rasch den Tod herbeiführt.» ${ }^{35}$

Dans une troisième série d'expériences, Zahn teste, à la suite de Carl Binz ${ }^{36}$, l'effet de la quinine sur l'émigration des globules blancs. Il constate que cet effet est différent selon le mode d'application du produit:

«Die Localapplication des Chinin. muriat. retardiert und hemmt die Auswanderung nur sehr wenig, bewirkt aber ein sofortiges Absterben der ausgewanderten weissen Blutkörperchen.

Subcutane oder innerliche Anwendung desselben Mittels kann in Folge von Kreislaufstörungen die Auswanderung verhindern, oder doch lange Zeit zurückhalten und dann verringern; die weitere Folge davon ist aber in den meisten Fällen der Tod.» ${ }^{37}$

Voici donc cette thèse résumée. Apporte-t-elle les réponses attendues par Klebs? Permet-elle d'affirmer que le Microsporon septicum est la cause d'une maladie infectieuse?

Klebs et Zahn répondent affirmativement: ${ }^{38}$ l'action du champignon n'est cependant pas isolée; d'autres microorganismes pourraient également posséder ce pouvoir ${ }^{39}$ une action chimique, dépendante ou non du Microsporon, n'est par ailleurs pas exclue.

Dans sa critique, Felix Birch-Hirschfeld ${ }^{40}$ reconnaît à ce travail une grande qualité: celle d'être l'une des premières études expérimentales portant sur la recherche des facteurs étiologiques de la suppuration; il lui est cependant reproché de ne pas comporter des expérimentations de contrôle utilisant des irritants chimiques. Les conclusions de Zahn, fondées sur les prémisses de Klebs (qui ont elles-mêmes fait l'objet d'une critique) ${ }^{41}$ sont, selon le commentateur, prématurées. 
Les points faibles de la démonstration de Zahn apparaissent en toute clarté en 1878, lorsque Robert Koch (1843-1910) publie un ouvrage intitulé Untersuchungen über die Ätiologie der Wundinfektionskrankheiten ${ }^{42}$ : la recherche de l'étiologie d'une maladie infectieuse exige pour ce dernier une clarification préalable des postulats méthodologiques de même que la mise en œuvre de conditions techniques particulières. ${ }^{43}$ Prouver qu'un microorganisme est la cause d'une infection revient pour Koch à prouver sa qualité infectieuse spécifique: il s'agit donc de démontrer que la bactérie incriminée est dans tous les cas, le seul agent causal; ${ }^{44}$ la méthode qui consiste à seulement observer les effets de l'inoculation d'un microorganisme sur les tissus (comme l'a fait Klebs), n'exclut pas une autre cause, telle par exemple une intoxication. ${ }^{45}$ L'identification de l'agent infectieux ${ }^{46}$ est complémentaire à l'étude des altérations anatomo-pathologiques spécifiques qu'il provoque. ${ }^{47}$

Nous voilà ainsi parvenus au terme de notre investigation. Nous sommes maintenant en mesure d'évaluer l'intérêt historique de la recherche de Zahn. Il réside dans la nature de l'argumentation utilisée par cet auteur dans la controverse des années 1870 sur la cause de la suppuration.

L'analyse que j'ai proposée montre que l'ensemble de la discussion peut se réduire à un débat sur la définition de la cause d'une infection. Selon Klebs, la présence du Microsporon septicum est toujours accompagnée d'une suppuration locale ainsi que d'une septicémie; cette dernière est le fidèle témoin d'une infection de l'organisme. Zahn confirme expérimentalement que l'inoculation de ce microorganisme provoque une inflammation qui peut secondairement se généraliser: la suppuration locale et la septicémie sont donc les deux pôles d'un même processus infectieux auquel le Microsporon septicum a donné naissance. Cette démonstration est suffisante aux yeux des deux pathologues pour considérer ce microorganisme comme la cause de l'infection. ${ }^{48}$ En opposition à ce point de vue, Koch, en tant que bactériologiste, n'accorde à un microorganisme le statut de cause que si ce dernier, une fois identifié puis isolé, est la condition sine qua non, c'est-à-dire dans son vocabulaire, la cause spécifique de l'infection. ${ }^{49}$ C'est ce que la thèse de Zahn n'est précisément pas parvenue à démontrer.

L'influence de Koch se fait très tôt sentir: en 1880, Alexander Ogston ${ }^{50}$ établit qu'un agent spécifique, une bactérie du genre micrococcus, est la cause la plus fréquente de la suppuration. ${ }^{51}$ Le développement de la bactériologie a ainsi donné lieu à une nouvelle lecture de la théorie de l'inflammation de Cohnheim. ${ }^{52}$ 
Si l'analyse de la thèse de Zahn que j'ai proposée ne nous autorise pas à conférer à ce travail des lettres de noblesse, elle nous permet du moins de mettre en lumière le rôle joué par le futur professeur genevois au sein d'un débat aux enjeux multiples, qui a mobilisé la plupart des esprits scientifiques à une époque charnière: la période qui a précédé le développement conjoint de la science bactériologique et de l'étude morphologique différentielle des diverses formes étiologiques de l'inflammation. ${ }^{53}$

\section{Notes}

1 D'autres microorganismes peuvent plus rarement être la cause d'un phénomène suppuratif: E. Coli, Aerobacter aerogenes, Proteus, Bacillus pyocyaneus, de même que les meningocoques et les gonocoques ont cette faculté; voir à ce sujet le manuel de Robbins Stanley and Angell Marcia, Basic Pathology, Philadelphia, 1971, p.46-47, de même que le livre de Bulloch William, The History of Bacteriology, London 1938 (reprint 1960), p. 149-151.

2 Consulter les chapitres «Eiter», «Eiterung» et «Entzündung» dans les Schmidt's Jahrbücher édités par H.E. Richter et A. Winter, des années 1868 à 1872, de même que les chapitres «Entzündung und Eiterung» dans les Jahresberichte über die Leistungen und Fortschritte in der gesammten Medicin, édités par R. Virchow et A. Hirsch, des années 1869 à 1873.

3 Consulter l'article de Birch-Hirschfeld Felix, Die neuern pathologisch-anatomischen Untersuchungen über krankmachende Schmarotzerpilze, in Schmidt's Jahrbücher, 155, 1872, p. 97-109.

4 Zahn Friedrich Wilhelm, Untersuchungen über Thrombose. Bildung der Thromben, in Archiv für pathologische Anatomie und Physiologie und für klinische Medicin, 62, 1875, p. $81-124$.

5 Zahn Friedrich Wilhelm, Über die Folgen des Verschlusses der Lungenarterien und Pfortaderäste durch Embolie, in Verhandlungen der Gesellschaft Deutscher Naturforscher und Arzte, Leipzig, 1898, zweiter Teil, zweite Hälfte, p. 9-11.

6 Zahn Friedrich Wilhelm, Über einige anatomische Kennzeichen der Herzklappeninsufficienzen, in Verhandlungen des XIIIten Kongresses für innere Medizin, 1895, p. 351.

7 Zahn a été appelé à occuper ce poste à l'instigation de Carl Vogt, alors recteur de cette université nouvellement fondée.

Il n'existe pas à ma connaissance d'ouvrage biographique sur Zahn. Les articles nécrologiques anonymes parus dans la Revue médicale de la Suisse romande, 24, 1904, p.621-624, et dans le Münchener medizinischen Wochenschrift, 51, 1904, p. 1694, ont été consultés. 
8 Les documents biographiques consultés sont les suivants: Ernst Paul und Klebs Arnold C., Edwin Klebs, in Verhandlungen der deutschen Pathologischen Gesellschaft, 17.Tagung, München, 1914, p.588-597; Ernst Paul, Edwin Klebs, in Münchener medizinische Wochenschrift, 61, 1914, p. 193-196 et 251-254; Haberling W., Klebs Edwin, in Biographisches Lexikon der hervorragenden Arzte, zweite Auflage, Berlin und Wien, 1931, Bd.III, p.539-540; Roethlin Otto Mario, Edwin Klebs (1834-1913). Ein früher Vorkämpfer der Bakteriologie und seine Irrfahrten, Zürich, 1962.

9 Schmidt's Jahrbücher, 155, 1872, p. 97.

10 La doctrine du pléomorphisme a longtemps été défendue par le botaniste zurichois Carl von Nägeli (1817-1891). L'histoire de cette doctrine est exposée dans Bulloch William, op. cité p. 178, 188, 202 et 385. Consulter également à ce propos Kern Lilly, Deutsche Bakteriologie im Spiegel englischer medizinischer Zeitschriften, 1875-1885, Zürich (Z.M.A. Nr.116) 1977, p. 11.

11 Schmidt's Jahrbücher, 155, 1872, p. 98-102 et p. 108-109.

12 Billroth Theodor, Neue Beobachtungsstudien über Wundfieber, in Archiv für klinische Chirurgie, 13, 1872, p. 647-667; voir en particulier p. 658-660.

13 Cohn Ferdinand, Untersuchungen über Bakterien, in Beiträge zur Biologie der Pflanzen, Bd. II, Heft 2, 1872, p. 126-224. Consulter également l'article «Bakterien» dans la RealEncyclopädie der gesamten Heilkunde, 4e éd. 1907, vol. II, p. 247-248.

14 Billroth Theodor, Untersuchungen über die Vegetationsformen von Coccobacteria septica und den Antheil, welchen sie an der Entstehung und Verbreitung der accidentellen Wundkrankheit haben, Berlin, 1874. C'est dans ce livre que cette algue est pour la première fois décrite.

15 L'article «suppuration» du Dictionnaire encyclopédique des sciences médicales (Dechambre), 1889, rédigé par Albert Henocque, contient un compte rendu détaillé sur la question. Une revue historique plus récente du débat est exposée dans Bulloch William, op. cité p. 129-152.

16 Ogston Alexander, Über Abscesse, in Archiv für klinische Chirurgie, 25, 1880, p. 588-600.

17 Consulter Von Recklinghausen Friedrich Daniel, Über Eiter und Bindegewebskörperchen, in Archiv für pathologische Anatomie und Physiologie und für klinische Medicin, 28, 1863, p. 157-197. Cohnheim se fonde sur les résultats de cet article lors de ses propres recherches sur l'inflammation publiées en 1867. (Voir la note suivante.)

18 Cohnheim Julius, Über Entzündung und Eiterung, in Archiv für pathologische Anatomie und Physiologie und für klinische Medicin, 40, 1867, p. 1-79.

19 Nous ne pouvons développer ce sujet dans le cadre de cet article. Nous renvoyons le lecteur aux articles suivants: Weber C. O., Zur Entwicklungsgeschichte des Eiters, in Archiv für pathologische Anatomie und für klinische Medicin, 15, 1858, p.465-530; Lubarsch Otto, Virchows Entzündungslehre und ihre Weiterentwicklung bis zur Gegenwart, in Virchows Archiv, 235, 1921, p. 186-211; Diepgen Paul, Die Lehre von der Entzündung. Von der Begründung der Zellularpathologie bis zum Aufkommen der Bakteriologie, Wiesbaden, 1953; Rather L.J., Addison and the white corpuscules: an aspect of nineteenth century biology, Berkeley and Los Angeles, 1972.

20 Les caractéristiques morphologiques des globules blancs sont décrites dans: Donne Alfred, Cours de microscopie, Paris, 1844, p.81-85, et Donne Alfred, Cours de microscopie. Atlas, Paris, 1845, planche VI, fig. 20; consulter également Virchow Rudolf, Die Cellularpathologie in ihrer Begründung auf physiologische und pathologische Gewebelehre, Berlin, 1858, 
p. 125, 134-135, 141 (à comparer avec la p. 134); l'ouvrage de Boroviczeny K. YG., Schipperges H., Seidler E., Einführung in die Geschichte der Hämatologie, Stuttgart, 1974, p. 7476 , est éclairant à ce propos. Il est intéressant de noter que Virchow constate une identité morphologique entre les globules blancs et les cellules du pus dans les deux ouvrages suivants: Virchow Rudolf, Gesammelte Abhandlungen zur wissenschaftlichen Medicin, Frankfurt, 1856, p. 223 et 288 ainsi que dans Virchow Rudolf, 1858, op. cité p. 135 et 140. Ce point avait déjà été discuté avant Virchow: voir à ce propos l'article «Pus, pyogénie», rédigé par P.-H. Bérard dans le Dictionnaire de médecine, Paris, 1842, tome XXVI, p.411504, en particulier les p. 414-419.

21 Billroth Theodor, Mancherlei über die morphologischen Vorgänge bei der Enzündung, in Österreicher medizinische Jahrbücher, 18, 1869, p. 1-36.

L'auteur mentionne dans cet article les principales questions qui étaient alors préoccupantes: par quel mécanisme les globules blancs peuvent-ils traverser la paroi des vaisseaux sanguins? S'agit-il d'un phénomène transitoire ou durable? Pourquoi les globules blancs émigrent-ils?

22 Cohnheim Julius, 1867, op. cité p. 27, 62, 63.

23 Cohnheim s'en préoccupera ultérieurement ainsi qu'en témoignent de longs passages des Vorlesungen über allgemeine Pathologie, Berlin, 1877, vol. I, p.249-257, et des Vorlesungen über allgemeine Pathologie, Berlin, 1882, 2éd. vol. I, p. 287-304.

24 Consulter les chapitres «Entzündung und Eiterung» des Schmidt's Jahrbücher, des années 1867 à 1872.

25 Voir à ce propos Bulloch W. op. cité p. 129-141. L'exemple de Th. Billroth est intéressant: dans Billroth Theodor, Über die Verbreitung der entzündlichen Prozesse, in Sammlung klinischer Vorträge, herausgegeben von R. Volkmann, Leipzig, 1870, le chirurgien viennois suppose qu'un «poison phlogogène», (sur la nature duquel il ne se prononce pas) doit être impliqué dans la pathogénie de la suppuration diffuse (infectieuse) régulièrement observée en cas de plaies suppurées. Il avait déjà émis cette hypothèse en 1865 dans l'article suivant : Beobachtungsstudien über Wundfieber und accidentellen Wundkrankheiten, in Archiv für klinische Chirurgie, 6, 1865, p. 469. L'idée d'une substance «phlogogène» se trouve déjà chez Virchow (voir Gesammelte Abhandlungen zur wissenschaftlichen Medicin, Frankfurt, 1856, p. 703).

26 Klebs Edwin, Die Ursache der infectiösen Wundkrankheiten, in Correspondenz-Blatt für Schweizer Ärzte, 1, 1871, p.242.

27 Claude Bernard démontre en 1856 les propriétés du curare sur l'interruption de la transmission des impulsions du neurone moteur au muscle volontaire. Consulter à ce propos: Bernard Claude, Analyse physiologique des propriétés des systèmes musculaires et nerveux au moyen du curare, in Comptes rendus de l'Académie des Sciences, (Paris), 43, 1856, p. 825-829.

28 Zahn Friedrich Wilhelm, Zur Lehre von der Entzündung und Eiterung, Heidelberg, 1871, p. $13,16,18,19$.

29 Ibid., p. 19.

30 Lister Joseph, On a New Method of Treating Compound Fracture, Abscess, etc., with Observations on the Conditions of Suppuration, in Lancet, 1, 1867, p. 326-329, 357-359, 387-389, 507-509, et Lancet, 2, 1867, p. 95-96; et Lister Joseph, On the Antiseptic Principle in the Practice of Surgery, in Lancet, 2, 1867, p. 353-356, 668-669. 
31 Zahn, 1871, op. cité, p.52.

32 La méthode de filtration, mise au point par Zahn, est décrite en détail par Klebs dans le Correspondenz-Blatt für Schweizer Ärzte, 1, 1871, p. 277, ainsi que dans Tiegel Ernst, Ủber die Fiebererregende Eigenschaft des Microsporon septicum, Bern, 1871, p. 16-17. Tiegel était, en même temps que Zahn, un élève de Klebs.

33 Zahn, 1871, op. cité p.52.

34 Le filtrat est obtenu par une nouvelle filtration à travers de la terre d'argile (Zahn, 1871, op. cité, p. 21).

35 Ibid, p. 52.

36 Binz Carl, Pharmakologische Studien über Chinin, in Archiv für pathologische Anatomie und Physiologie und für klinische Medicin, 46, 1869, p.67-105, et 129-168. Ce travail démontre l'action inhibitrice de la quinine sur l'émigration des globules blancs.

37 Zahn, 1871, p. 52.

38 Klebs, 1871, op. cité, p. 246, et Zahn, 1871, op. cité, p. 4, 20 et 52.

39 Zahn, 1871, op. cité, p. 16, 18, 19, 31, 52.

40 Schmidt's Jahrbücher, 1872, op. cité, p. 102-103, et p. 108-109.

41 Voir la note $n^{\circ} 11 \mathrm{du}$ présent article.

42 Koch cite dans cet ouvrage le travail publié par Klebs en 1872: Beiträge zur pathologischen Anatomie der Schusswunden, en lequel il reconnait le premier essai d'établir une relation causale entre des bactéries et des maladies infectieuses consécutives à des plaies (Koch 1878, op. cité, p. 10). Il démontre quelques pages plus loin que cet essai ne fut cependant pas concluant.

43 Koch, 1878, op. cité, p. 15, 22, 27, 29-39. Koch s'exprime de la manière suivante: «Fassen wir das, was als thatsächlich Bekanntes zusammengestellt wurde, und die daran geknüpften Erörterungen kurz zusammen, so kommen wir zu dem Ergebniss, dass die zahlreichen Befunde von Mikroorganismen bei Wundinfectionskrankheiten und die damit im Zusammenhang stehenden experimentellen Untersuchungen die parasitische Natur dieser Krankheiten wahrscheinlich machen, dass ein vollgültiger Beweis dafür bis jetzt noch nicht geliefert ist und auch nur dann geschafft werden kann, wenn es gelingt, die parasitischen Mikroorganismen in allen Fällen der betreffenden Krankheit aufzufinden, sie ferner in solcher Menge und Vertheilung nachzuweisen, dass alle Krankheitserscheinungen dadurch ihre Erklärung finden, und schliesslich für jede einzelne Wundinfectionskrankheit einen morphologisch wohl characteristischen Mikroorganismus als Parasiten festzustellen» (p.27). La coloration à l'aniline des préparations selon la méthode de Weigert (p. 30), permet une meilleure identification des éléments observés que la méthode proposée en 1871 par F.D. von Recklinghausen (p.29). Il faut cependant pour obtenir de bons résultats utiliser un microscope muni d'un condensateur performant tel par exemple celui qu'a inventé Ernst Abbé et qu'a construit Carl Zeiss (p. 34-39) : l'ouvrage de Hans Peter Nowak, Geschichte des Mikroskops, Zürich, 1984, p.42-44, nous donne les renseignements de détail nécessaires à ce sujet.

44 Koch, 1878, op. cité, p. 22, 27 et 74-77.

45 Ibid., p. 15.

46 Ibid., p. 70-71. Koch ne parvient pas à identifier l'agent causal de l'infection dans cet ouvrage. C'est Alexander Ogston qui y parviendra deux ans plus tard en se fondant sur les postulats et la technique de Koch (voir la note $n^{\circ} 16$ du présent article). 
47 Koch, 1878, op. cité, p. 69.

48 Consulter à ce sujet l'article de Codell Carter K., Edwin Klebs' Criteria for Disease Causality, in Medizinhistorisches Journal, 22, 1987, p. 80-89, en particulier les p. 81, 82, 84 et 87-89. L'auteur estime que Klebs recourt à ce type de démonstration à plusieurs reprises dans son œuvre.

49 Un logicien dirait que le microorganisme est pour Koch la cause nécessaire de l'infection: voir à ce propos King Lester S., The Philosophy of Medicine, Cambridge and London, 1978, chap. 9, p. 212. Il est intéressant de relever que la notion de nécessité (logique) implique chez Koch celle de spécificité (biologique) lorsqu'elle s'applique à une interrelation pathologique entre deux organismes vivants individualisés. Une étude plus approfondie du rapport qu'entretenaient au $19^{\mathrm{e}}$ siècle les concepts de cause spécifique et de cause nécessaire pourrait éclairer, me semble-t-il, l'étude des relations entre la bactériologie et la pathophysiologie à cette époque.

50 Voir Bulloch William, 1938, op. cité p. 149.

51 Voir Ogston Alexander, 1880, op. cité p. 599.

52 Koch avait déjà exercé une influence sur Cohnheim lors de sa visite à l'Institut de pathologie de Breslau en 1875 : voir Diepgen Paul, 1953, op. cité p. 83. Dans les Vorlesungen über allgemeine Pathologie, Berlin, 1877, vol. I, p. 249-257, Cohnheim consacre un chapitre aux inflammations infectieuses («infectiöse Entzündungen») auxquelles il attribue une cause bactérienne (voir en particulier la p. 256.)

53 Consulter à ce propos l'ouvrage suivant: Aschoff L., Kuester E., Schmidt W. J., Hundert Jahre Zellforschung (Protoplasma-Monographien Band 17), Berlin, 1938, p. 241-248.

\section{Summary}

\section{The contribution of Friedrich Wilhelm Zahn (1845-1904) to the study of inflammation}

Prior to being named to the first chair of Pathology at Geneva University, Friedrich Wilhelm Zahn had published, in 1871, his doctoral thesis entitled Zur Lehre von der Entzündung und Eiterung. This work, written under the direction of Edwin Klebs, at Berne, demonstrated experimentally that the so-called Microsporon septicum (an infectious fungus identified by Klebs) provoked a local inflammation as well as remote secondary focal suppurations. This study apparently confirmed Klebs's hypothesis that Microsporon septicum, usually present in wounds, is the cause of an infectious disease. Zahn's research as well as the work of Klebs fell under attack: the method of identifying the microorganism as well as the demonstration of his causal relationship to inflammation were not conclusive. At the end of the discussion, it was 
admitted that the status of a cause of infection could be attributed to a microorganism only under the two following conditions : the latter must be identified with Koch's method, and his causal relationship to the infectious disease must be specific. This is precisely what Zahn's thesis was unable to prove.

The analysis of his work as well as of the critical arguments enables us to follow and understand the controversy on the cause of inflammation and suppuration at the dawn of the bacteriological age.

\section{Zusammenfassung}

\section{Der Beitrag von Friedrich Wilhelm Zahn (1845-1904) zur Entzündungsfor- schung}

Vor seiner Berufung auf den ersten Lehrstuhl für Pathologie an die Universität Genf veröffentlichte Friedrich Wilhelm Zahn 1871 seine Doktorarbeit Zur Lehre von der Entzündung und Eiterung. In dieser unter Edwin Klebs in Bern verfassten Studie konnte Zahn experimentell zeigen, dass das sogenannte Microsporon septicum (ein von Klebs identifizierter infektiöser Pilz) sowohl eine örtliche Entzündung als auch eine entfernte, sekundäre, herdförmige Eiterung verursachte. Dies bestätigte die Hypothese von Klebs, wonach das Microsporon septicum eine Wundinfektionskrankheit auslöse. Zahns Untersuchung gab Anlass zu Kritik, die sich auch gegen Klebs richtete: Die Identifizierung des Mikroorganismus und die Darlegung des Kausalzusammenhangs seien nicht schlüssig. Am Ende der Auseinandersetzung wurde anerkannt, dass ein infektiöser Krankheitszustand nur dann auf einen Mikroorganismus zurückgeführt werden dürfe, wenn folgende zwei Bedingungen erfüllt seien: jener muss nach Kochs Methode identifiziert werden, und der Kausalzusammenhang mit der Infektionskrankheit muss spezifisch sein. Gerade dies aber hatte Zahn nicht zu beweisen vermocht.

Die Analyse seiner Untersuchung gestattet uns ein besseres Verständnis der verschiedenen Argumente, die in der Auseinandersetzung um die Ursache der Entzündung und Eiterung am Beginn der bakteriologischen Aera vorgebracht wurden.

Lazare Benaroyo, Dr. med.

Medizinhistorisches Institut der Universität Zürich

Rämistrasse 71

CH-8006 Zürich 